\title{
Implementation and Verification of High-Order Accurate Discontinuous Galerkin Method on 2-D unstructured grids
}

\author{
Wengeng Zhao ${ }^{a}$, Hongwei Zheng ${ }^{\mathrm{b}}$ \\ LHD, Institute of Mechanics, Chinese Academy of Science, No.15 Beisihuanxi Road, Beijing \\ 100190, China \\ azhaowengeng@imech.ac.cn, bh.zheng@imech.ac.cn
}

Keywords: High-Order Accuracy, Discontinuous Galerkin, Data limiting, Unstructured grids

\begin{abstract}
In this paper, the discontinuous Galerkin method (DG) is applied to solve the 2D Euler equation. DG can be easily used in the unstructured girds, which has advantages in dealing with problems with complex boundaries. High order accuracy is achieved by higher order polynomial approximations within elements. In order to capture the shock without oscillation, the limiter is also applied. The performance of DG is illustrated by three numerical experimental tests, which show the potential of DG in engineering applications. The vortex propagation problem is to verify high-order accuracy of DG, while Sob problem and forward step problem are used to illustrate the ability to capture shock.
\end{abstract}

\section{Introduction}

The discontinuous Galerkin method (DG) was introduced by Reed and Hill in the framework of neutron transport and successively analyzed by Lesaint and Raviart for the linear advection equation [1]. It has been proposed since 1980s. Cockburn and Shu came up with the Runge-Kutta discontinuous Galerkin methods during the 90 s of the twenty century, which has showed good performance when solving the problems with discontinuity and has been extended to hydrodynamics, aerodynamics and wave propagation etc [2].

In this work we applied DG to solve the 2D Euler equation in the unstructured girds. The outline of this paper is structured as follows. The DG algorithm for Euler equation under unstructured grids is given in Section 2. Different forms of base and the relationship between the Gaussian integral points and accuracy are described. Numerical experiments are discussed in Section 3. Section 4 is devoted to the conclusions.

\section{Discontinuous Galerkin Formulation}

We consider the two-dimensional Euler equations which can be written into the form as follows

$$
\frac{\partial \boldsymbol{u}}{\partial t}+\frac{\partial \boldsymbol{f}}{\partial x}+\frac{\partial \boldsymbol{g}}{\partial y}=0 \quad \boldsymbol{r}=(x, y) \in \Omega
$$

The conservative variables $\boldsymbol{u}_{\text {and }}$ the Cartesian components $\boldsymbol{f}_{\text {and }} \boldsymbol{g}$ of the inviscid (Euler) flux function $\boldsymbol{F}$ are given by

$$
\boldsymbol{u}=\left(\begin{array}{c}
\rho \\
\rho u \\
\rho v \\
E
\end{array}\right), \quad \boldsymbol{f}=\left(\begin{array}{c}
\rho \\
\rho u^{2}+p \\
\rho u v \\
u(E+p)
\end{array}\right), \quad \boldsymbol{g}=\left(\begin{array}{c}
\rho \\
\rho u v \\
\rho v^{2}+p \\
v(E+p)
\end{array}\right) .
$$

where $\rho$ is the fluid density, $u$ and $v$ are the velocity components, $p$ is the pressure, and $E$ is the total energy per unit volume. By assuming that the fluid obeys to the perfect gas state equation, p can be computed by 


$$
p=(\gamma-1)\left(E-\frac{1}{2} \rho\left(u^{2}+v^{2}\right)\right) .
$$

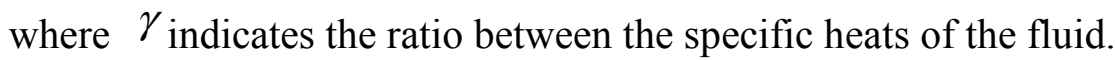

Multiplying Eq.1 by a "test function" $\varphi$, integrating over the domain $\Omega$, and performing integration by parts we obtain the weighted residual formulation

$$
\int_{\Omega} \varphi \boldsymbol{u}_{t} d V+\oint_{\partial \Omega} \varphi \boldsymbol{F} \cdot \boldsymbol{n} d S-\int_{\Omega} \nabla \boldsymbol{\varphi} \cdot \boldsymbol{F} d V=0
$$

Space Discretization The computational domain is subdivided into non-overlapping triangular elements. By applying Eq.4 to each element $T_{i}$, we can obtain the discrete analogue on the computational grid.

Assuming that both solution and test function can be expressed by polynomial approximation and denoting the polynomial basis as $\boldsymbol{\xi}(\boldsymbol{r})=\left\{\xi_{1}(\boldsymbol{r}), \ldots, \xi_{n}(\boldsymbol{r})\right\}^{T}$. By admitting only the functions $\boldsymbol{u}_{i}(\boldsymbol{r}, t)$ and $\varphi_{h}$ and letting $\boldsymbol{U}^{i}=\left\{Q_{i}^{1}, \ldots, Q_{i}^{n}\right\}^{T}$, the equation can be further written as

$$
\frac{d \boldsymbol{U}^{i}}{d t}+\left(M^{i}\right)^{-1}\left(\oint_{\partial T_{i}} \boldsymbol{\xi} \boldsymbol{F} \cdot \boldsymbol{n} d S-\int_{T_{i}} \nabla \boldsymbol{\xi} \cdot \boldsymbol{F} d V\right)=0 .
$$

Analyzing Eq.5, it's easy to find that three integral parts need to be determined during the space discretization [5].

It's common practice in the finite element method to deal with the real triangle element by introducing a reference element $\hat{T}_{i}$ in a nondimensional space and a geometric transformation which maps the reference element onto the real elements in the physical space. What's more, when mapping the real triangle element into the reference, the Jacobian matrix is a constant, by which the computational time can be reduced enormously. We use the area coordinates to get the nondimensional transfer. $\mathrm{P}$, located in the element, area coordinates of which are $\left(L_{1}, L_{2}, L_{3}\right)$. The corresponding parameters can be found in [6]

By using the expression of the area coordinates, the test functions of different order of accuracy are showed in [6].

Next, the three integration parts are evaluated separately.

1) $I_{1}=\iint_{\Omega} \xi_{i} \xi_{i} d \Omega$

The mass matrix in element $T_{i}$, denoted by $I_{1}$, is symmetric and has no connection with the variables of the flow field. It can be obtained by the integration of the test function directly.

2) $I_{2}=\oint_{\partial T_{i}} \xi_{j} \boldsymbol{F} \cdot \boldsymbol{n} d S=\sum_{r=1}^{K} \int_{A_{r}} \xi_{j} \boldsymbol{F} \cdot \boldsymbol{n} d S$

The corresponding relation between the demanding accuracy of $I_{2}, I_{3}$ and the minimum integral points in triangular can be summarized in Table 1. On edge $r$, we can obtain the final expression of Gaussian numerical integral [7]

$$
\int_{A_{r}} \xi_{j} \boldsymbol{F} \cdot \boldsymbol{n} d S \approx \sum_{r}^{K} \sum_{s=1}^{n s} w_{r s} \xi_{j}\left(\boldsymbol{r}_{r s}\right) \hat{\boldsymbol{F}}\left(u^{L}\left(\boldsymbol{r}_{r s}\right), u^{R}\left(\boldsymbol{r}_{r s}\right), \boldsymbol{n}_{r}\right) A_{r} .
$$

where $\mathrm{K}$ denotes the number of side, ns denotes the number of integral points on one side, $w_{r s}$ denotes the weights of Gaussian point, $\boldsymbol{r}_{r s}$ denotes the Gaussian points. 
Table 1 Relation between the demanding accuracy of $I_{2}, I_{3}$ and the minimum integral points in triangular

\begin{tabular}{c|c|c|c|c|c}
\hline $\begin{array}{c}\text { Order of } \\
\text { accuracy }\end{array}$ & $\begin{array}{c}\text { Order of } \\
\text { basis }\end{array}$ & $\begin{array}{c}\text { Demanding } \\
\text { order of } I_{2}\end{array}$ & $\begin{array}{c}\text { No. of integral } \\
\text { points in } I_{2}\end{array}$ & $\begin{array}{c}\text { Demanding } \\
\text { order of } I_{3}\end{array}$ & $\begin{array}{c}\text { No. of integral } \\
\text { points in } I_{3}\end{array}$ \\
\hline 2 & 1 & 3 & $2 \times 3=6$ & 2 & 3 \\
3 & 2 & 5 & $3 \times 3=9$ & 4 & 6 \\
4 & 3 & 7 & $4 \times 3=12$ & 6 & 12 \\
5 & 4 & 9 & $5 \times 3=15$ & 8 & 16 \\
\hline
\end{tabular}

Due to the discontinuous function approximation, flux terms are not uniquely defined at element interfaces. It is at this stage that the technique traditionally used in the FVM schemes is borrowed by the DG. The flux function $\boldsymbol{F}\left(\boldsymbol{u}_{h}\right) \cdot \boldsymbol{n}$ is replaced by numerical flux function $\hat{\boldsymbol{F}}\left(\boldsymbol{u}_{h}^{-}, \boldsymbol{u}_{h}^{+}\right)$. The numerical function $\hat{\boldsymbol{F}}\left(\boldsymbol{u}_{h}^{-}, \boldsymbol{u}_{h}^{+}\right)$depends on the internal state of the boundary $\boldsymbol{u}_{h}^{-}$, on the neighboring element state of the boundary $\boldsymbol{u}_{h}^{+}$, and the norm vector of the side $\boldsymbol{n}$.

Several common numerical flux functions satisfying the above criteria can be used, such as Godunov, Lax-Friedrichs, Roe, Engquist-Ohser, HLLE flux function. Here we adopt the Lax-Friedrichs flux function [8]

3) $I_{3}=\int_{T_{i}} \nabla \xi_{j} \cdot \boldsymbol{F} d V$

Expression of the numerical integration of $I_{3}$ are as follows

$$
\int_{T_{i}} \nabla \xi_{j} \cdot \boldsymbol{F} d V \approx A_{i} \sum_{s=1}^{n v} w_{s} \nabla \xi_{j}\left(\boldsymbol{r}_{s}\right) \boldsymbol{F}\left(\boldsymbol{U}_{i}\left(\boldsymbol{r}_{s}\right)\right) .
$$

where $n v, \boldsymbol{r}_{s}, w_{s}$, denotes the number of integral points, Gaussian point, weights of Gaussian point separately.

Now, we can figure out $I_{3}$.All of the above work makes the space discretization available and we will illustrate how to discretize term involving in time.

Time Discretization The equations defining the approximate solution can be rewritten in ODE form as in Eq.6. The time integration of the semidiscrete system is accomplished by means of an explicit multi-stage third-order TVD (total variation diminishing) Runge-Kutta scheme advised by Cockburn and Shu in [9].

Limiter To enhance the stability of the method and eliminate possible spurious oscillations in the approximate solution, date limiter should be introduced. The local slope limiting [10] is practical and effective in applications and has shown high quality in many experimental tests.

In our test cases, three different boundaries need to be handled. The detail can be found in [11] of how to distinguish each of the boundary conditions and how to handle them separately.

\section{Numerical Tests}

In this section, we present several numerical results with the scheme discussed above.

Vortex Propagation Problem The vortex propagation problem is a typical case to conform the accuracy of different methods [7]. In Table 3, we list a number of results, showing the global error at the final time as a function of the number of elements, $\mathrm{K}$, and the order of the local approximation, N. Results under different orders are compared and the high-order results on coarse grid is the same as the low order results on fine grids.

The high accuracy reflected in Table 2 comes at a price. The CPU time increases rapidly with the increase of the accuracy. It may look as if high order accuracy is not quite appealing to us, but a closer look at the result above will reach the contrary conclusion. We take the $L_{2}$ error for example. To make the result more clear, we list the correspond results in Table 3. From the Table 3, it can be easily found that the execution time decreases when using high order accuracy. A high-order method is superior in terms of efficiency. 
Table 2 Errors for propagation vortex case at $t=0.5$ using the linear, square and cubic polynomial basis on the triangular grids

\begin{tabular}{lllllllll}
\hline $\begin{array}{l}\text { Order of } \\
\text { accuracy }\end{array}$ & $\begin{array}{l}\text { Cell } \\
\text { Number }\end{array}$ & $L_{1}$ error & $L_{1}$ order & $L_{2}$ error & $L_{2}$ order & $L_{\infty}$ error & $L_{\infty}$ order & $\begin{array}{c}\text { CPU } \\
\text { time }\end{array}$ \\
\hline \multirow{2}{*}{} & $\mathrm{K}$ & & $\mathrm{N}$ & & $\mathrm{N}$ & & $\mathrm{N}$ & $\mathrm{T}$ \\
\hline & 236 & $2.555 \mathrm{e}-3$ & - & $7.049 \mathrm{e}-3$ & - & $6.119 \mathrm{e}-2$ & - & 0.6 \\
& 928 & $6.913 \mathrm{e}-4$ & 1.81 & $1.901 \mathrm{e}-3$ & 1.71 & $1.579 \mathrm{e}-2$ & 1.89 & 3.6 \\
& 3690 & $1.715 \mathrm{e}-4$ & 2.01 & $4.720 \mathrm{e}-4$ & 2.01 & $3.812 \mathrm{e}-3$ & 2.05 & 31.5 \\
\hline 3 & 236 & $4.939 \mathrm{e}-4$ & - & $1.166 \mathrm{e}-3$ & - & $8.845 \mathrm{e}-3$ & - & 1.7 \\
& 928 & $6.923 \mathrm{e}-5$ & 2.83 & $1.659 \mathrm{e}-4$ & 2.81 & $9.270 \mathrm{e}-4$ & 3.25 & 10.4 \\
& 3690 & $1.064 \mathrm{E}-5$ & 2.70 & $2.613 \mathrm{e}-5$ & 2.67 & $1.783 \mathrm{e}-4$ & 2.38 & 82.9 \\
\hline 4 & 236 & $7.294 \mathrm{e}-5$ & - & $1.756 \mathrm{e}-4$ & - & $2.116 \mathrm{e}-3$ & - & 5.2 \\
& 928 & $5.189 \mathrm{e}-6$ & 3.81 & $1.313 \mathrm{e}-5$ & 3.74 & $1.010 \mathrm{e}-4$ & 4.39 & 31.9 \\
& 3690 & $4.270 \mathrm{e}-7$ & 3.60 & $7.944 \mathrm{e}-7$ & 4.05 & $6.352 \mathrm{e}-6$ & 4.00 & 257.0 \\
\hline
\end{tabular}

Table $3 L_{2}$ error and CPU time of different order

\begin{tabular}{ccc}
\hline Order of accuracy & $L_{2}$ error & CPU time \\
\hline 2 & $4.720 \mathrm{e}-4$ & 31.5 \\
3 & $1.659 \mathrm{e}-4$ & 10.4 \\
4 & $1.756 \mathrm{e}-4$ & 5.2 \\
\hline
\end{tabular}

2D Sob Problem The Sob problem with initial discontinuity is studied here to test the performance of DG to capture the discontinuity. As has been discussed elaborately in the data limiting part, the local slope limiter has to be employed to obtain smooth result. Computation was carried out at different grids and the results at the $\mathrm{t}=0.2$ are compared with the exact solution, seeing in Fig.1. The right-running shock waves, contact discontinuity, and the left-running expansion wave can be distinguished clearly. In addition, the slope limiter can prevent the spurious oscillation efficiently.

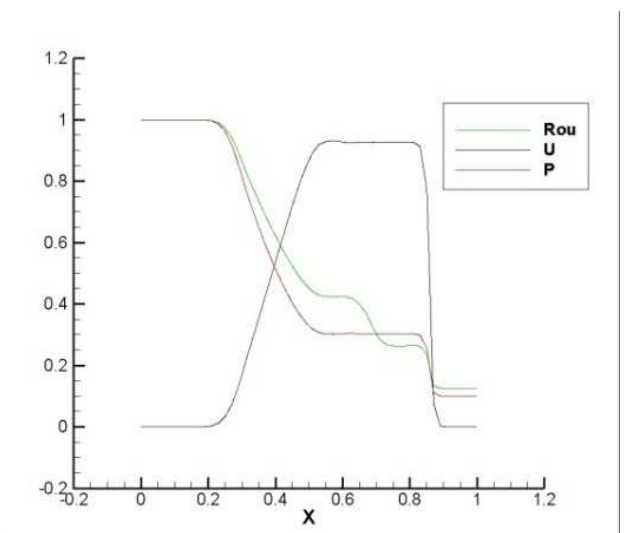

(a) $\mathrm{dx}=0.02$

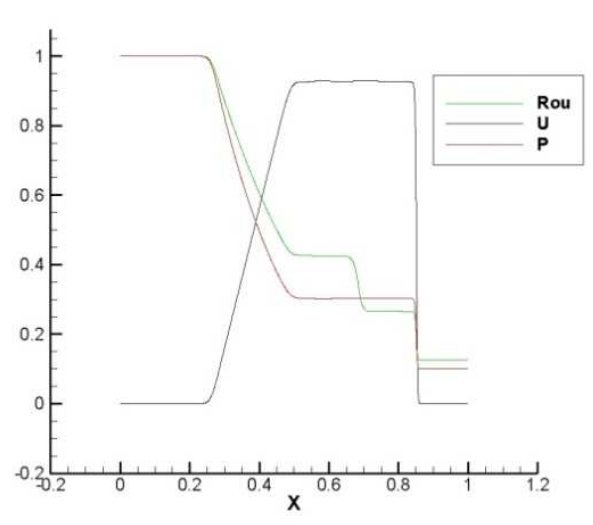

c) $\mathrm{dx}=0.005$

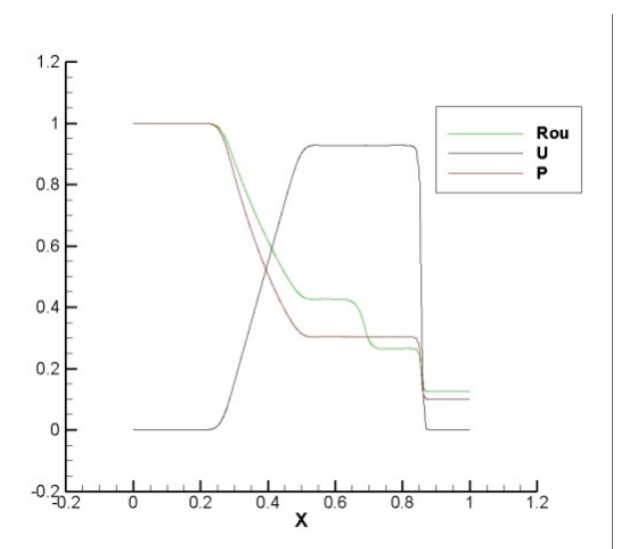

(b) $\mathrm{dx}=0.01$

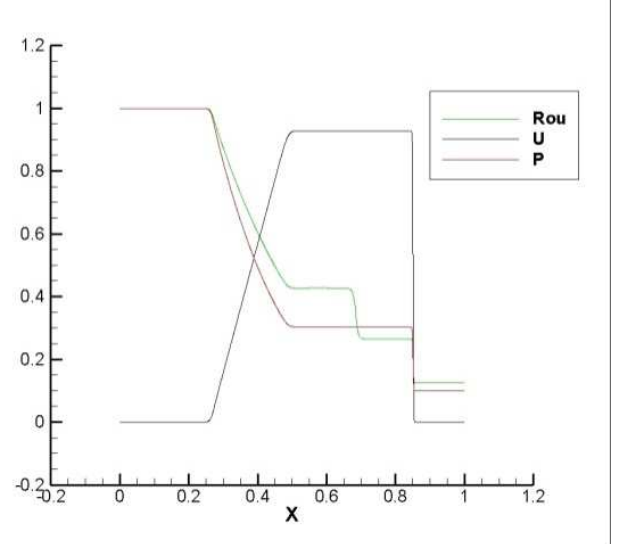

d) $\mathrm{dx}=0.0025$

Fig. 1 Results at $\mathrm{t}=0.2$ in different girds using the linear basis ( $d x$ denotes the size of the cell) 
Flow past a Forward Facing Step. A right-going Mach 3 uniform flow enters a wind tunnel. Reflective boundary conditions are applied along the wall of the tunnel and inflow and outflow boundary conditions are applied at the left-hand side and the right-hand side, respectively.

The result at $\mathrm{t}=4$ are shown in Fig.3. There's high similarity between the result with the scheme of DG and that with other popular scheme. Bow shock right ahead of the step, Mach reflection on the upper wall and the shock interaction in the middle of the flow field can be recognized explicitly. Besides, the interface of the shock wave is quite sharp and the solution is smooth at the region without shock wave.

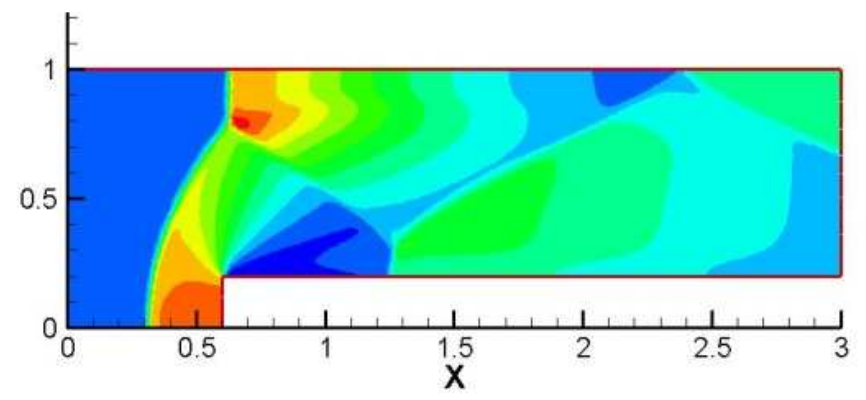

Fig.3 Density cloud of the forward-facing step problem

\section{Summary}

In this paper, we have systematically investigated the cell-centered high-order accurate discontinuous Galerkin method and stated how to apply this method on two-dimensional unstructured triangular grids in detail. DG is locally conservative, stable, and high-order accurate methods which can easily handle complex geometries. Three experimental cases are used to evaluate the performance of DG and the results are quite satisfactory. In the vortex propagation problem, the high-order accuracy is verified using linear, square and cubic element. In the Sob problem and the forward step problem, the shock is calculated without any oscillation. These shows that DG is a promising method and becoming an alternative method to the standard finite volume method in the engineering applications, for example, in turbo machinery, magneto hydro dynamics, chemical transport, multi-phase fluids.

\section{References}

[1] F. Bassi and S. Rebay; A High-Order Accurate Discontinuous Finite Element Method for the Numerical Solution of the Compressible Navier-Stokes Equations. Journal of Computational Physics 131,267-279, (1997)

[2] HE Li-xing, ZHANG Lai-ping, ZHANG Han-xin: Discontinuous Galerkin finite element method on 3D arbitrary elements, Acta Aerodynamica Sinica, Vol.25, (2007)

[3] LI Xi-le, YANG Yong, ZHANG Dong-yun; Research on Discontinuous Galerkin Method for Two Dimensional Euler Equations on Structured Grids. Aeronautical Computing Technique. Vol. 39(2009)

[4] YAN Chao, YU Jian: On the Achievements And Prospects for the Methods of Computational Fluids Dynamics. Advances in Mechanics Vol. 41(2011)

[5] Wang Gang, Xu Heyong, Ye Zhengyin: Integration Strategy for High-Order Discontinuous Galerkin Method for Solving Euler Equations, Journal of Northwestern Polytechnical University, Vol.29(2011)

[6] Wang Xucheng, Finite Element Method, Tsinghua University Press, Beijing, 2003

[7] Yuzhi Sun Z.J. Wang, Evaluation of discontinuous Galerkin and spectral volume methods for scalar and system conservation laws on unstructured grids. International Journal for numerical methods in fluids. 2004, 45 
[8] YU Xi-jun, ZHOU Tie: Discontinuous Finite Element Methods for Solving Hydrodynamic Equations. Chinese Journal of Computational Physics, Vol. 22 (2005)

[9] Cockburn B, Shu C W: TVB Runge-Kutta local projection discontinuous Galerkin finite element method for conservation laws II : General Framework. Mathematics of Computation, (1989)

[10] Cockburn B, Shu C W: TVB Runge-Kutta discontinuous Galerkin method for conservation laws V: Multidimensional systems. Journal of Computational Physics (1998)

[11] J. Blazek: Computational Fluid Dynamics: Principles And Applications, Elservier, (2001) 
Design and Manufacture in Mechanical Engineering

10.4028/www.scientific.net/AMM.432

Implementation and Verification of High-Order Accurate Discontinuous Galerkin Method on 2-D Unstructured Grids

10.4028/www.scientific.net/AMM.432.221 